\title{
A Polycomb group protein complex with sequence-specific DNA-binding and selective methyl-lysine-binding activities
}

\author{
Tetyana Klymenko, ${ }^{1}$ Bernadett Papp, ${ }^{1}$ Wolfgang Fischle, ${ }^{2,3}$ Thomas Köcher, ${ }^{1}$ Malgorzata Schelder, ${ }^{1}$ \\ Cornelia Fritsch, ${ }^{1}$ Brigitte Wild, ${ }^{1}$ Matthias Wilm, ${ }^{1}$ and Jürg Müller ${ }^{1,4}$ \\ ${ }^{1}$ Gene Expression Programme, European Molecular Biology Laboratory, 69117 Heidelberg, Germany; ${ }^{2}$ Laboratory of \\ Chromatin Biology, The Rockefeller University, New York, New York 10021, USA
}

Polycomb response elements (PREs) are specific cis-regulatory sequences needed for transcriptional repression of HOX and other target genes by Polycomb group (PcG) proteins. Among the many PcG proteins known in Drosophila, Pho is the only sequence-specific DNA-binding protein. To gain insight into the function of Pho, we purified Pho protein complexes from Drosophila embryos and found that Pho exists in two distinct protein assemblies: a Pho-dINO80 complex containing the Drosophila INO80 nucleosome-remodeling complex, and a Pho-repressive complex (PhoRC) containing the uncharacterized gene product dSfmbt. Analysis of PhoRC reveals that $\mathrm{dSfmbt}$ is a novel PcG protein that is essential for HOX gene repression in Drosophila. PhoRC is bound at HOX gene PREs in vivo, and this targeting strictly depends on Pho-binding sites. Characterization of dSfmbt protein shows that its MBT repeats have unique discriminatory binding activity for methylated lysine residues in histones $\mathrm{H} 3$ and $\mathrm{H} 4$; the MBT repeats bind mono- and di-methylated $\mathrm{H} 3-\mathrm{K} 9$ and $\mathrm{H} 4-\mathrm{K} 20$ but fail to interact with these residues if they are unmodified or tri-methylated. Our results establish PhoRC as a novel Drosophila PcG protein complex that combines DNA-targeting activity (Pho) with a unique modified histone-binding activity (dSfmbt). We propose that PRE-tethered PhoRC selectively interacts with methylated histones in the chromatin flanking PREs to maintain a Polycomb-repressed chromatin state.

[Keywords: Polycomb group; PRE; Pho/dYY1; MBT repeat; histone methylation]

Supplemental material is available at http://www.genesdev.org.

Received December 22, 2005; revised version accepted February 23, 2006.

The regulation of gene expression by Polycomb group (PcG) and trithorax group (trxG) proteins represents a paradigm for understanding the establishment and maintenance of heritable transcriptional states during development. PcG and trxG genes were first genetically identified as regulators that are required for the long-term maintenance of HOX gene expression patterns in Drosophila. PcG proteins keep HOX genes silenced in cells in which they must stay inactive, whereas trxG proteins maintain the active state of these genes in appropriate cells (for review, see Francis and Kingston 2001; Ringrose and Paro 2004). This regulatory relationship is conserved in vertebrates, where PcG and trxG proteins also regulate HOX gene expression. In addition, mammalian PcG

\footnotetext{
${ }^{3}$ Present address: Max Planck for Biophysical Chemistry, Laboratory of Chromatin Biochemistry, Am Fassberg 11, 37077 Goettingen, Germany. ${ }^{4}$ Corresponding author.

E-MAIL Juerg.Mueller@embl.de; FAX 49-6221-387518.

Article published online ahead of print. Article and publication date are at http://www.genesdev.org/cgi/doi/10.1101/gad.377406.
}

and trxG proteins have also been implicated in X-chromosome inactivation, hematopoietic development, control of cell proliferation, and oncogenic processes.

Drosophila HOX genes are among the best-studied target genes of the PcG/trxG system. Different studies have led to the identification of specific cis-regulatory sequences in HOX genes that are called Polycomb response elements (PREs) and are required for silencing by PcG proteins. PREs are typically several hundred base pairs in length, and they function as potent transcriptional silencer elements in the context of HOX reporter genes as well as in a variety of other reporter gene assays (e.g., Chan et al. 1994; Zink and Paro 1995; Sengupta et al. 2004). This operational definition of PREs is complemented by their classification as DNA sequences to which PcG proteins bind, directly or indirectly. Among the 14 cloned Drosophila PcG genes, only Pleiohomeotic (Pho) and Pho-like (Phol) encode sequence-specific DNAbinding proteins (Brown et al. 1998, 2003). Pho and Phol bind the same DNA sequence, and while the two proteins act to a large extent redundantly, double mutants 
show severe loss of HOX gene silencing (Brown et al. 2003). DNA-binding sites for Pho and Phol are present in all PREs that have been characterized to date, and mutational analyses of these binding sites have shown that they are essential for silencing by PREs (Brown et al. 1998, 2003; Mihaly et al. 1998; Fritsch et al. 1999; Shimell et al. 2000; Busturia et al. 2001; Mishra et al. 2001; Ringrose et al. 2003). In contrast, none of the other 12 characterized PcG proteins bind DNA in a sequencespecific manner. However, formaldehyde cross-linking studies showed that several of these proteins specifically associate with the chromatin of PREs in tissue culture cells and in developing embryos and larvae (Strutt and Paro 1997; Orlando et al. 1998; Cao et al. 2002). Biochemical studies revealed that most of these non-DNAbinding PcG proteins are components of either PRC1 or PRC2, two distinct PcG protein complexes that have recently been purified and characterized (Shao et al. 1999; Saurin et al. 2001; Cao et al. 2002; Czermin et al. 2002; Müller et al. 2002; Tie et al. 2003). Specifically, PRC1 contains the PcG proteins Polycomb (Pc), Posterior sex combs (Psc), Polyhomeotic (Ph), Sex combs extra/Ring (Sce/Ring), and Sex combs on midleg (Scm), whereas PRC2 contains the three PcG proteins Extra sex combs (Esc), Enhancer of zeste $[E(z)]$, and Suppressor of zeste 12 [Su(z)12] (Shao et al. 1999; Saurin et al. 2001; Czermin et al. 2002; Müller et al. 2002).

What is the role of Pho and Phol at PREs? Biochemically purified PRC1 and PRC2 do not contain Pho or Phol ( $\mathrm{Ng}$ et al. 2000; Saurin et al. 2001; Müller et al. 2002). Several recent studies investigated possible physical interactions between Pho and PRC1 or PRC2 complex components. Based on coimmunoprecipitation and GST pull-down assays, it was proposed that Pho directly interacts with several different PRC1, PRC2, and SWI/ SNF complex components (Poux et al. 2001; Mohd-Sarip et al. 2002; Wang et al. 2004). However, on polytene chromosomes of phol; pho double mutants, the binding of PRC1 and PRC2 to HOX genes and at most other loci is largely unperturbed, suggesting that, at least in this tissue, Pho and Phol are not strictly required for keeping PRC1 and PRC2 anchored to HOX genes (Brown et al. 2003).

To gain insight into the biological function of Pho, we biochemically purified Pho-containing protein complexes from Drosophila. Our data show that Pho exists in two distinct multiprotein complexes that, contrary to expectation, do not contain any of the previously characterized PcG proteins. Our functional analysis of one of these Pho complexes that we name PhoRC provides evidence that its binding to PREs is required for maintaining repressive HOX gene chromatin.

\section{Results}

Drosophila INO80 complex and dSfmbt copurify with Pho

We used a tandem affinity purification (TAP) strategy (Rigaut et al. 1999) to purify Pho protein complexes from
Drosophila embryonic nuclear extracts. We constructed a transgene that expresses a TAP-tagged Pho fusion protein (Pho-TAP) under the control of the Drosophila $\alpha$-tubulin promoter and generated transgenic flies. To test whether the Pho-TAP protein is functional, we introduced the transgene into the genetic background of animals homozygous for $p h o^{1}$, a protein-negative allele of pho (Fig. 1A). pho ${ }^{1}$ homozygotes die as pharate adults, but they are rescued into viable and fertile adults that can be maintained as a healthy strain if they carry one copy of the transgene expressing Pho-TAP (Fig. 1A). The Pho-TAP protein can thus substitute for the endogenous Pho protein, and this shows that the fusion protein is functional.

We then purified proteins that are associated with the Pho-TAP protein from embryonic nuclear extracts, following the TAP procedure (Rigaut et al. 1999). Seven different polypeptides that consistently copurified with the Pho-TAP bait protein in several independent purifications were identified through sequencing of peptides from individual protein bands by nanoelectrospray tandem mass spectrometry (Fig. 1B; see Supplemental Material S1A for detailed information on mass spectrometry analysis). In addition to Pho, the isolated protein assembly contains the product of CG31212, a protein that is most closely related to yeast INO80, the SWI/SINF2-like nucleosome-remodeling subunit in the yeast INO80 complex (Shen et al. 2000). We shall therefore refer to the CG31212 locus as dINO80. Five other subunits of the Pho complex were identified as Reptin (Rept), Pontin (Pon), Actin (Act), and the two actin-related proteins dArp5 and dArp8, which are encoded by CG7940 and CG7846, respectively (Fig. 1B). These five proteins represent the Drosophila homologs of five core subunits that assemble together with INO80 to form the yeast INO80 complex (Shen et al. 2000). Specifically, Rept and Pont are homologs of the yeast Rvb1 and Rvb2 AAAATPases that constitute a DNA helicase in the INO80 complex. Act, dArp5, and dArp8 are homologs of the Actin, Arp5, and Arp8 proteins, respectively, that are present in the yeast INO80 complex. Thus, it appears that a Drosophila dINO80 complex copurifies with Pho. In addition, the purified material also contained the product of CG16975, a protein that is not conserved in yeast but is closely related to the product of the murine Scm-related gene containing four mbt domains (Sfmbt) (Usui et al. 2000); we therefore refer to the CG16975 gene as $d S f m b t$. The characteristic features of mammalian Sfmbt and the Drosophila dSfmbt protein are four malignant brain tumor (MBT) repeats and a sterile $\alpha$ motif (SAM) domain (Fig. 1D). The Drosophila genome encodes two other proteins that contain MBT repeats and show a similar domain architecture, 1/3) mbt and the PcG repressor Scm (Fig. 1D). Taken together, these findings suggest that Pho exists in multiprotein assemblies that contain a dINO80 complex and dSfmbt but, unexpectedly, none of the previously characterized PcG proteins.

We next analyzed the purified material by Western blot. As expected, antibodies against dSfmbt (see Materials and Methods) and antibodies against Rept and Pon 

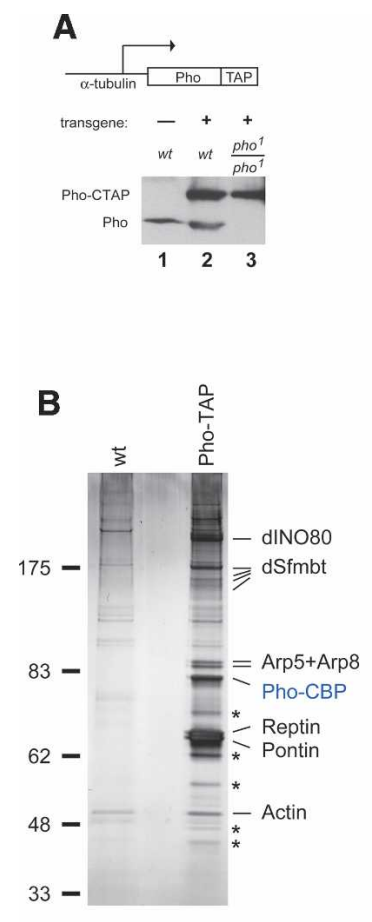
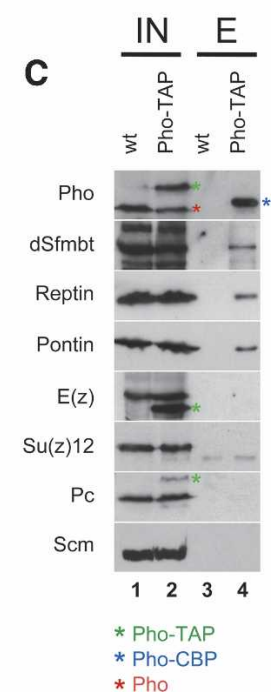

D

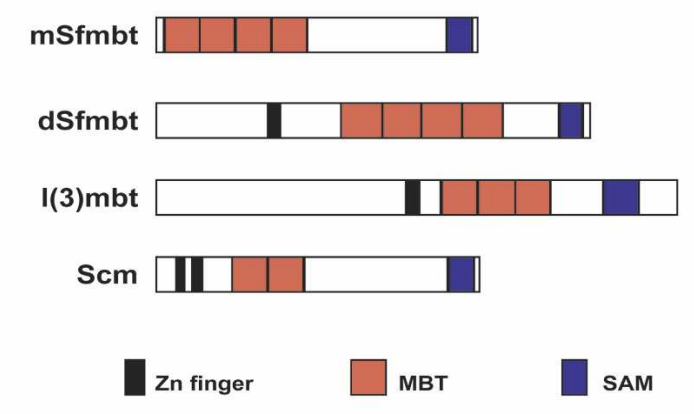

MBT repeats

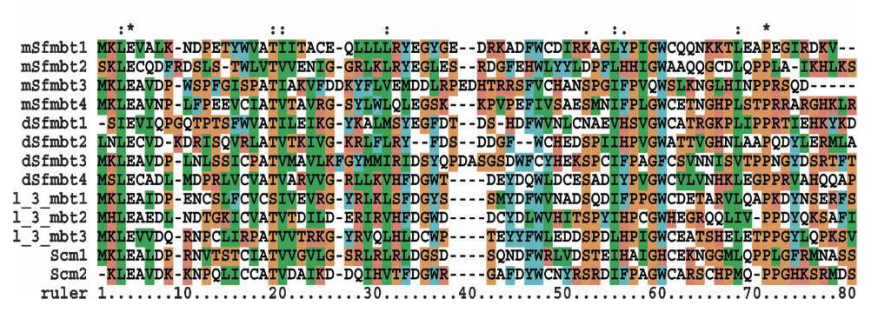

Figure 1. TAP of Pho protein complexes from Drosophila embryos. (A) Western blot of total embryo extracts from wild-type (lanes 1,2) or $p h o^{1} /$ pho $^{1}$ mutant embryos (lane 3) that carry the Pho-TAP transgene as indicated, probed with anti-Pho antibody. Note, pho ${ }^{1}$ is a protein-null allele. Stoichiometry of Pho and Pho-TAP protein cannot be compared since Pho antibody binds to Pho epitope and the protein A moiety in the Pho-TAP protein. $(B)$ Pho protein complexes purified from nuclear extract prepared from wild-type (wt) or Pho-TAP, pho $/$ pho $^{1}$ (Pho-TAP) Drosophila embryos visualized by silver staining. Input material for mock-purification from wild-type embryos and for purification from Pho-TAP embryos was normalized by protein concentration. Equivalent amounts of calmodulin-affinity resin was boiled in SDS sample buffer, and eluted material was separated on a $4 \%-12 \%$ polyacrylamide gel. The indicated proteins consistently copurified with Pho-CBP protein in several independent experiments; copurification of these proteins with Pho-CBP was independent of the genetic background (i.e., $p h o^{+}$or $p h o^{1} / p h o^{1}$ ). (Pho-CBP) Fusion protein containing the calmodulin-binding moiety of the TAP tag. Lower-molecular-weight dSfmbt bands were identified by microsequencing, and they likely correspond to dSfmbt degradation products. Asterisks indicate bands for which we have not been able to obtain unambiguous peptide sequence data; see Supplemental Material S1A for information on additional proteins that were identified by mass spectrometry. $(C)$ Western blot analysis of total embryonic nuclear extract input material (IN, lanes 1,2) from wild-type (wt) and Pho-TAP transgenic embryos and material eluted from calmodulin affinity resin (E, lanes 3,4) after purification. All panels come from the same batch of input material, and the eluates were all from the same batch of material purified from wild-type and Pho-TAP embryos, respectively. Note that the Pho-TAP embryos used for this experiment were $p h o^{+}$, but the same results were obtained if complexes were purified from Pho-TAP, pho $1 / p^{1} o^{1}$ embryos. Asterisks indicate endogenous Pho (red), Pho-CBP (blue), and Pho-TAP (green) protein; in lane 2, Pho-TAP is also detected by other antibodies due to protein A tag. Note signals for dSfmbt, Reptin, and Pontin but a lack of signals for $\mathrm{E}(z), \mathrm{Su}(z) 12, \mathrm{Pc}$, and Scm in lane 4. (D) Domain architecture of the three Drosophila MBT repeat proteins dSfmbt, 1(3) $\mathrm{mbt}$, and Scm and alignment of the corresponding MBT repeats. The mouse Sfmbt protein (mSfmbt) as described by Usui et al. (2000) is shown for comparison.

(Bauer et al. 2000) specifically detect bands of the expected size in the complex purified from Pho-TAP embryos but not in the material mock-purified from wildtype embryos (Fig. 1C). Antibodies against dINO80 (see Materials and Methods) also detect specific enrichment of the dINO80 protein (Supplementary Fig. S1B). In contrast, when we used antibodies against the PRC2 subunits $\mathrm{E}(\mathrm{z})$ or $\mathrm{Su}(z) 12$ or against the PRC1 subunits Pc or $\mathrm{Scm}$, we were unable to detect specific signals in the material that copurified with Pho, although each of these four proteins is readily detected in the nuclear extract used for purification (Fig. 1C). Thus, we find no evidence that PRC1 or PRC2 subunits would stably associate with Pho protein complexes.

\section{dSfmbt and dINO80 are present in two distinct Pho protein complexes}

Since the yeast genome does not contain any dSfmbtrelated protein, we wondered whether dSfmbt and dINO80 are part of distinct Pho protein complexes. To this end, we fractionated crude embryonic nuclear extracts by glycerol gradient sedimentation and probed individual fractions by Western blotting with antibodies against Pho, Pho-like, dINO80, and dSfmbt. Our results show that dINO80 and dSfmbt are present in separate fractions of the gradient but that Pho and Pho-like are present in both dINO80- and dSfmbt-containing fractions (Fig. 2A; data not shown). dSfmbt and dINO80 thus 
A

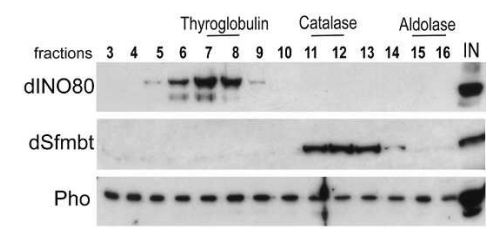

B

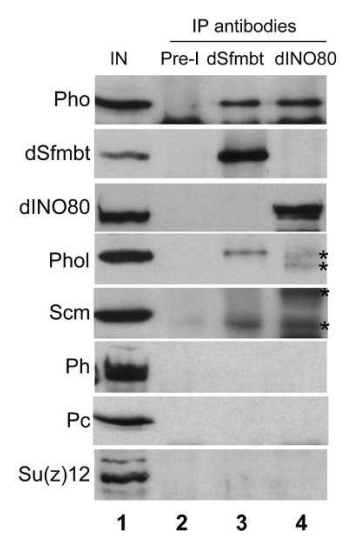

Figure 2. dSfmbt and dINO80 exist in distinct Pho protein complexes. (A) Crude embryonic nuclear extracts were separated on a $20 \%-50 \%$ glycerol gradient. Fractions were probed with antibodies against dINO80, dSfmbt, and Pho; (IN) nuclear extract input material. Thyroglobulin $(669 \mathrm{kDa})$, catalase $(232$ $\mathrm{kDa})$, and aldolase (158 kDa) were fractionated on a separate gradient that was run in parallel, and fractions containing these proteins are indicated on top. Note that dINO80 and dSfmbt are present in distinct complexes, both of which contain Pho; the presence of Pho in other fractions suggests that Pho may also exist in additional complexes. Pho-like is present across the whole gradient similarly to Pho (not shown). (B) Immunoprecipitations from embryonic nuclear extracts were probed with antibodies indicated on the left. (Lane 1) Nuclear extract input material (IN, 10\%) used for immunoprecipitation with preimmune serum (Pre-I, lane 2) or with antibodies against dSfmbt (lane 3) or dINO80 (lane 4). Pho is present in samples immunoprecipitated with dSfmbt or dINO80 antibodies, but dINO80 is not detected in dSfmbt precipitates, and dSfmbt is absent from dINO80 precipitates. Pho-like is enriched in samples immunoprecipitated with dSfmbt, but background bands in dINO80 IP reactions (asterisks) do not permit unambiguous detection of Pho-like protein. Note the absence of $\mathrm{Scm}, \mathrm{Ph}, \mathrm{Pc}$, or $\mathrm{Su}(z) 12$ in both dSfmbt and dINO80 immunoprecipitates; asterisks in the Scm Western blot panel indicate nonspecific bands.

exist in distinct protein complexes in embryonic nuclear extracts. It should be noted that Pho and Pho-like are also present in fractions that do not contain dINO80 or dSfmbt (Fig. 2A; data not shown). This suggests that Pho and Pho-like also exists in soluble protein assemblies that are distinct from the complexes that we identified here but that these assemblies are not stable enough to be isolated as complexes in our purification scheme.

To further investigate whether we purified two distinct Pho complexes, we next used antibodies against dSfmbt or dINO80 in coimmunoprecipitation assays. Pho protein was significantly enriched in both anti-dSfmbt and anti-dINO80 immunoprecipitated samples
(Fig. 2B). In striking contrast, we find that dINO80 is not detected in the anti-dSfmbt immunoprecipitated samples and that dSfmbt is not detected in the samples immunoprecipitated with anti-dINO80 antibodies (Fig. 2B). Taken together, these observations strongly suggest that dSfmbt and dINO80 are part of two distinct Pho protein complexes.

In Drosophila, Pho and the related Pho-like protein are functionally redundant (Brown et al. 2003). We therefore asked, whether Pho-like is also associated with dSfmbt and/or dINO80. We find that Pho-like is enriched in anti$\mathrm{dSfmbt}$ immunoprecipitates but the analysis of dINO80 immunoprecipitates provided no evidence for strong enrichment of Pho-like (Fig. 2B). We also note that we have been unable to detect Pho-like in the material purified from Pho-TAP embryos. This indicates that the interaction of dSfmbt with Pho and Pho-like is mutually exclusive.

As described above, we found no evidence that the purified Pho complexes contain PRC1 or PRC2 subunits. We next explored the possibility that Pho complex components such as dINO80 or dSfmbt might associate with PRC1 or PRC2 (e.g., as "adaptors") but that these interactions were disrupted during the Pho-affinity purification. We therefore tested whether PRC1 or PRC2 subunits could be coimmunoprecipitated with dSfmbt or with dINO80. We first analyzed anti-dSfmbt immunoprecipitated material for enrichment of Scm and Ph proteins. Like dSfmbt, the $\mathrm{Scm}$ and $\mathrm{Ph}$ proteins contain a C-terminal SAM domain. Previous in vitro assays suggested that the SAM domains of Scm and Ph mediate homo- and heterotypic interactions between these two proteins (Peterson et al. 1997). Yet, we failed to detect either $\mathrm{Scm}$ or $\mathrm{Ph}$ in anti-dSfmbt immunoprecipitated samples (Fig. 2B). Furthermore, we could not detect a dSfmbt signal in samples immunoprecipitated with either $\mathrm{Scm}$ or Ph antibodies (data not shown). Thus, we find no evidence for an interaction between dSfmbt and $\mathrm{Ph}$ or Scm under our experimental conditions. We also could not detect a $\mathrm{Ph}$ signal in anti-Scm precipitated samples, and we only observed a very weak Scm signal in anti-Ph precipitated material (data not shown; but note that Peterson et al. [2004] recently reported the same observations for coimmunoprecipitation reactions using antibodies against $\mathrm{Scm}$ and $\mathrm{Ph}$ ). In addition, we analyzed anti-dSfmbt immunoprecipitated samples for presence of $\mathrm{Pc}$, another PRC1 subunit, and for the presence of the PRC2 subunit Su(z)12. Neither of these two proteins was detected in the immunoprecipitated material (Fig. 2B). Finally, we note that we could not detect $\mathrm{Scm}, \mathrm{Ph}, \mathrm{Pc}$, or $\mathrm{Su}(z) 12$ in anti-dINO80 immunoprecipitates (Fig. 2B). In summary, these data suggest that Pho exists in two distinct complexes, a Pho-dSfmbt and a Pho-dINO80 complex.

\section{Pho and dSfmbt are bound to PREs in vivo}

We next asked whether components of the purified Pho complexes are associated with PREs in vivo. To this end, we performed chromatin immunoprecipitation (X-ChIP) 
assays. Drosophila embryos were treated with formaldehyde and DNA that was cross-linked to Pho, dSfmbt, dINO80, Reptin, Pontin, or Ph was immunoprecipitated with antibodies against these proteins. Real-time quantitative PCR was used to measure the abundance of the following endogenous and transgene PREs in the immunoprecipitates. The bxd and iab-7 PREs in the HOX genes Ultrabithorax $(U b x)$ and Abdominal-B $(A b d-B)$, respectively, are well-characterized, and Pho binds to these PREs in vitro and in vivo (Chan et al. 1994; Hagstrom et al. 1997; Fritsch et al. 1999; Mishra et al. 2001; Wang et al. 2004). We previously reported that $P R E_{D}$ a 572-bp core fragment of the bxd PRE, silences a $U b x$ $L a c Z$ reporter gene in imaginal discs and in embryos but that point mutations in all six Pho protein-binding sites in this fragment $\left(P R E_{D}\right.$ pho mut $)$ completely abolish its silencing capacity (Fritsch et al. 1999). We therefore performed X-ChIP assays in transformed embryos that carried either the wild-type $P R E_{D}$ or the mutated $P R E_{D}$ pho mut reporter gene; this allowed us to directly compare protein binding at the transgenic PRE with protein binding at the endogenous $b x d$ and iab-7 PREs in the same preparation of chromatin. Specific PCR primer sets allowed us to distinguish X-ChIP signals at the reporter gene PRE from signals at the endogenous bxd PRE (Fig. 3). We found that $\mathrm{Pho}, \mathrm{Ph}$, and, importantly, also dSfmbt are specifically bound at the endogenous $b x d$ and iab-7 PREs but not at sequences flanking those PREs (Fig. 3). In contrast, we were unable to detect binding of dINO80, Reptin, or Pontin at any of the sequences analyzed (data not shown). Pho, dSfmbt, and $\mathrm{Ph}$ are also bound at the $P R E_{D}$ fragment in the transgene, but, strikingly, binding signals of Pho, dSfmbt, and Ph are severely reduced at the mutated $P R E_{D \text { pho mut }}$ fragment (Fig. 3). Taken together, these data show that Pho-dSfmbt complexes are bound at PREs in vivo and that binding of these complexes to PREs requires DNA-binding sites for Pho. Since we have been unable to detect association of dINO80 complex components with PREs in this assay, we focused our further analysis on the characterization of Pho-dSfmbt complexes; in-depth characterization of Pho-dINO80 complexes will be the subject of a different study.

\section{Reconstitution of Pho-dSfmbt and Pho-like-dSfmbt complexes}

We next asked whether Pho-dSfmbt complexes could be reconstituted from recombinant proteins. We used baculovirus vectors to coexpress the two proteins in Sf9 cells. Flag-affinity purification from extracts of Sf9 cells that express Pho-Flag and untagged dSfmbt resulted in the isolation of a dimeric Pho-dSfmbt complex (Fig. 4A, lane 3). We refer to this dimeric complex as Pho-repressive complex (PhoRC). Notably, PhoRC is stable in buffers containing up to $2 \mathrm{M} \mathrm{KCl}$ (Fig. 4A). The interaction between Pho and dSfmbt appears to be specific, because Flag-affinity purification from cells coexpressing dSfmbt and Flag-Esc or from cells coexpressing dSfmbt and FlagPc resulted in the isolation of single Flag-Esc or Flag-Pc protein, respectively (Fig. 4A, lanes 8,9 ). In addition, we
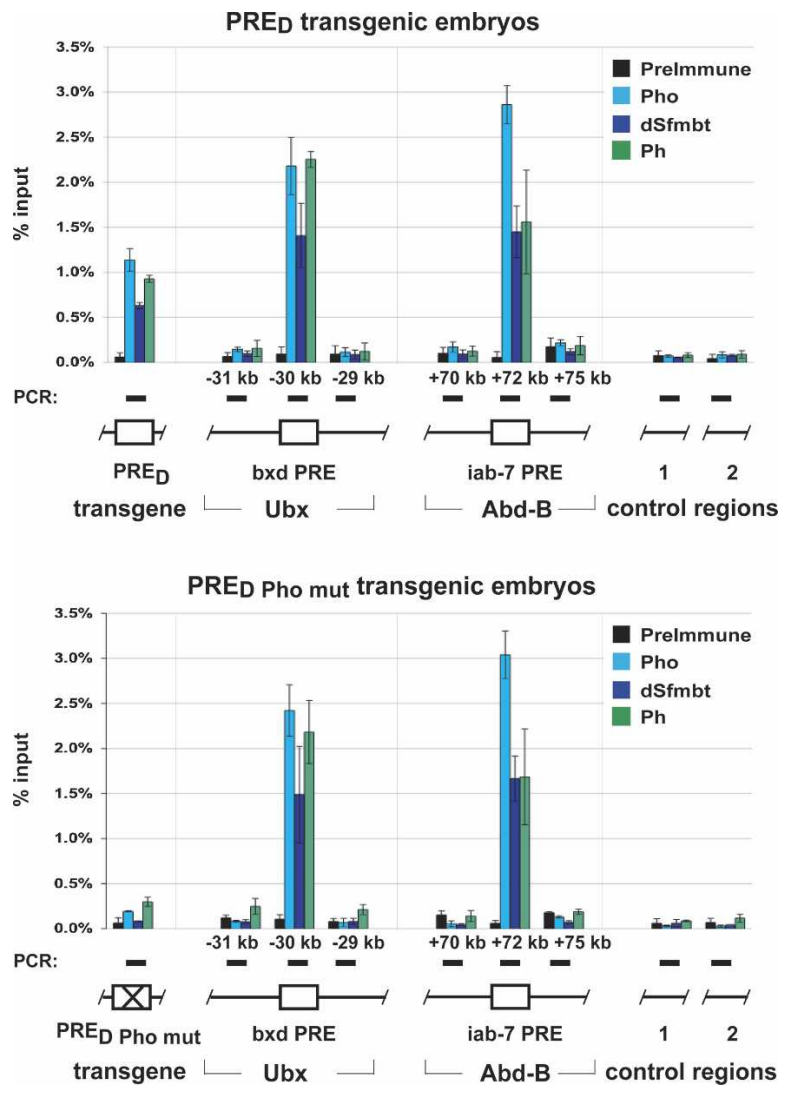

Figure 3. Binding of dSfmbt to PREs depends on Pho proteinbinding sites. (Top) X-ChIP analysis on $P R E_{D}$ transformant embryos. Pho, dSfmbt, and $\mathrm{Ph}$ are specifically bound at the $b x d$ and $i a b-7$ PREs in the $U b x$ and $A b d-B$ loci, respectively, and to the wild-type $b x d$ PRE in the $P R E_{D}$ reporter gene (Fritsch et al. 1999). The location of PREs with respect to the $U b x$ or $A b d-B$ transcription start site is indicated in kilobases. No binding is detected in regions flanking the PREs and at two intergenic locations elsewhere in the genome. The embryos carried a single copy of the $P R E_{D}$ transgene. (Bottom) X-ChIP analysis on $P R E_{D}$ Pho mut transformant embryos. Binding of Pho, dSfmbt, and $P h$ at $P R E_{D \text { Pho mut }}$ is strongly reduced due to mutation of all six Pho protein-binding sites in the $P R E_{D}$ Pho mut fragment (Fritsch et al. 1999). Binding signals at the endogenous bxd and iab-7 PREs is comparable in chromatin from $P R E_{D}$ and from $P R E_{D \text { Pho mut }}$ embryos. X-ChIP signals are represented as the fraction $(\%)$ of input material precipitated in each IP reaction; signals represent results from immunoprecipitation reactions performed on three independently purified batches of chromatin.

found that upon coexpression of dSfmbt, Pho, Reptin, and Pontin in various combinations, we could only purify either PhoRC or Reptin-Pontin complexes (Fig. 4A, lanes 6,7; data not shown). These observations suggest that Pho does not bind to Reptin or Pontin and that the association between Pho and the dINO80 complex therefore must be mediated by interaction with a different subunit of the dINO80 complex.

We then asked whether dSfmbt also forms stable complexes with Pho-like. Flag-affinity purification from cells coexpressing dSfmbt and Flag-Pho-like resulted in the isolation of a dSfmbt-Pho-like complex that was stable 


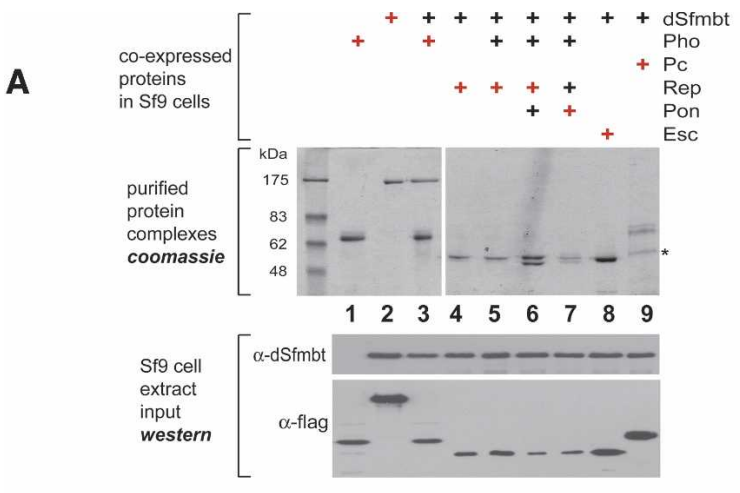

B

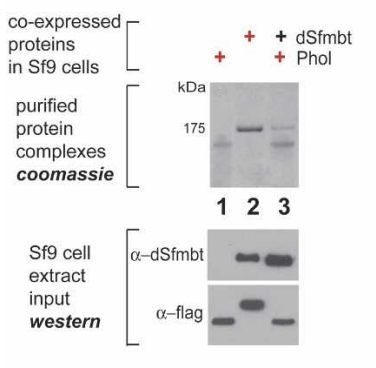

\section{C}

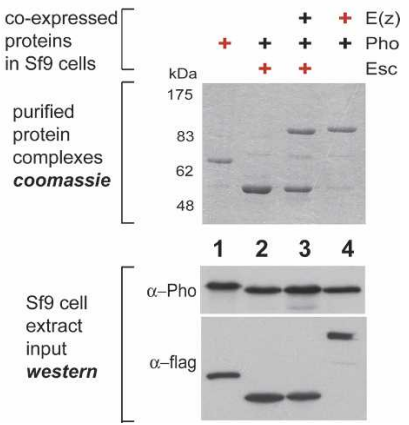

Figure 4. Reconstitution of recombinant PhoRC. (Layout in $A-C$, top) Indicated Flag-tagged (red + ) and untagged (black + ) proteins were (co)expressed in Sf9 cells, affinity-purified via the Flag tag, separated by SDS-PAGE, and visualized by coomassie staining. (Below) Western blot of corresponding Sf9 total cell extracts prior to purification to reveal coexpression of proteins at comparable levels. (A) dSfmbt and Pho form a stable complex. (Top) Purified PhoRC contains comparable amounts of dSfmbt and Flag-Pho proteins (lane 3), but Sfmbt and Pho do not copurify with Flag-Reptin, Flag-Pontin, Flag-Esc, or Flag-Pc, respectively (lanes 4-9). (Lanes 6,7) As yeast Rvb1p/Rvb2p (Jonsson et al. 2004), Reptin and Pontin are expected to form a stable complex, and the presence of this complex thus provides an internal control for complex reconstitution. (Below) Western signals show that dSfmbt and Flag-tagged proteins were coexpressed at similar ratios in all experiments. $(B$, lane 3$) \mathrm{dSfmbt}$ and Pho-like form a stable complex that contains comparable amounts of both proteins. (C) Pho does not form stable complexes with Esc and/or E $(z)$. Note that Pho does not copurify either with Flag-Esc (lane 2) or with Flag-E(z) (lane 4) alone and that stoichiometric Flag-Esc-E $(z)$ complexes without Pho are purified if Flag-Esc, $\mathrm{E}(z)$, and Pho are coexpressed (lane 3).

in buffers containing up to $2 \mathrm{M} \mathrm{KCl} \mathrm{(Fig.} \mathrm{4B,} \mathrm{lane} \mathrm{3).}$ dSfmbt can thus form stable complexes with Pho as well as with Pho-like.

Finally, we asked whether Pho could form stable complexes with PRC2 subunits. The incentive for these experiments was recent studies that reported direct physical interactions between Pho and PRC2 subunits Esc and $\mathrm{E}(z)$ in GST pull-down assays (Wang et al. 2004). When we performed Flag-affinity purification from cells coexpressing Flag-Esc, E(z), and Pho, we could isolate a stable dimeric Flag-Esc-E(z) complex as expected (Ketel et al. 2005; Nekrasov et al. 2005). However, we failed to detect Pho protein in the purified material (Fig. 4C, lane 3). We also found no evidence that Pho can form stable complexes with either Flag-Esc or Flag-E(z) alone (Fig. 4C, lanes 2,4$)$. We conclude that dSfmbt can form a stable dimeric complex with either Pho or Pho-like, but fails to stably interact with PRC2 (sub)complexes under the same assay conditions.

\section{$\mathrm{d}$ Sfmbt is a novel PcG gene needed for HOX gene silencing}

We next asked whether dSfmbt is required for silencing of HOX genes in Drosophila. Since no mutations in the dSfmbt gene have been described, we used a homologous recombination strategy (Gong and Golic 2003) to generate $d S f m b t^{1}$, a $d S f m b t$ knockout allele that does not produce dSfmbt protein (for details, see Fig. 5A; Supplementary Fig. S2; Materials and Methods). $d S f m b t^{1}$ homozygotes or animals that are transheterozygous for $d S f m b t^{1}$ and $D f(2 L) B S C 30$, a chromosomal deletion that removes $d S f m b t$ and several other transcription units, die as larvae with small imaginal discs (for details, see Materials and Methods). Imaginal discs are segment-specific primordia of proliferating tissue that will differentiate into segment-specific structures in the adult fly, according to the combination of HOX genes that is expressed in a particular disc. We previously showed that HOX genes become de-repressed outside of their normal expression domains in imaginal disc cell clones that are homozygous for PcG mutations (Beuchle et al. 2001). To test whether removal of $d S f m b t$ causes loss of HOX gene silencing, we induced clones of $d S f m b t^{1}$ homozygous cells in imaginal discs of $d S f m b t^{1}$ heterozygotes and analyzed these clones for misexpression of HOX genes $U b x$ and $S c r$, using antibodies against their protein products. $d S f m b t^{1}$ mutant clones were identified by the absence of a GFP-expressing marker gene. We find that $d S f m b t$ mutant clones show strong and widespread misexpression of Ubx and Scr protein when analyzed $96 \mathrm{~h}$ after clone induction (Fig. 5B). Importantly, this misexpression of HOX genes is as severe as the misexpression observed in clones of strong PcG mutants (Beuchle et al. 2001). We also examined the kinetics of de-repression in $d S f m b t$ mutant clones by analyzing clones 24,48 , and $72 \mathrm{~h}$ after clone induction. $U b x$ and $S c r$ are stably silenced until 48 $\mathrm{h}$ after clone induction, when misexpression first becomes detectable in a few cells in the mutant clones, but widespread misexpression is detected $72 \mathrm{~h}$ after clone induction (Fig. 5B; data not shown). The loss of HOX gene silencing after removal of dSfmbt late in development classifies $d S f m b t$ as a novel PcG gene in Drosophila.

We previously showed that Pho and Pho-like function redundantly to maintain HOX gene silencing (Brown et al. 2003). pho single mutants show only very subtle misexpression of HOX genes, and Pho-like single mutants show no HOX misexpression. In contrast, clones of cells that lack both Pho and Pho-like protein show severe mis- 
A

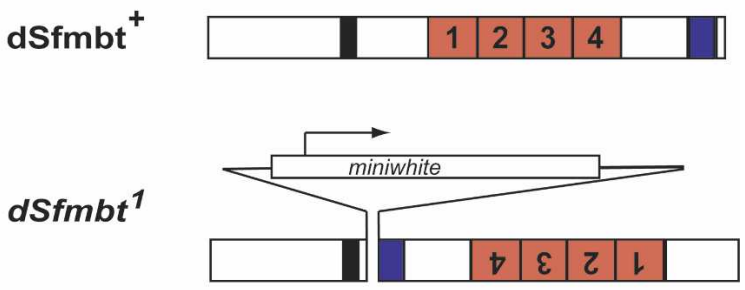

B

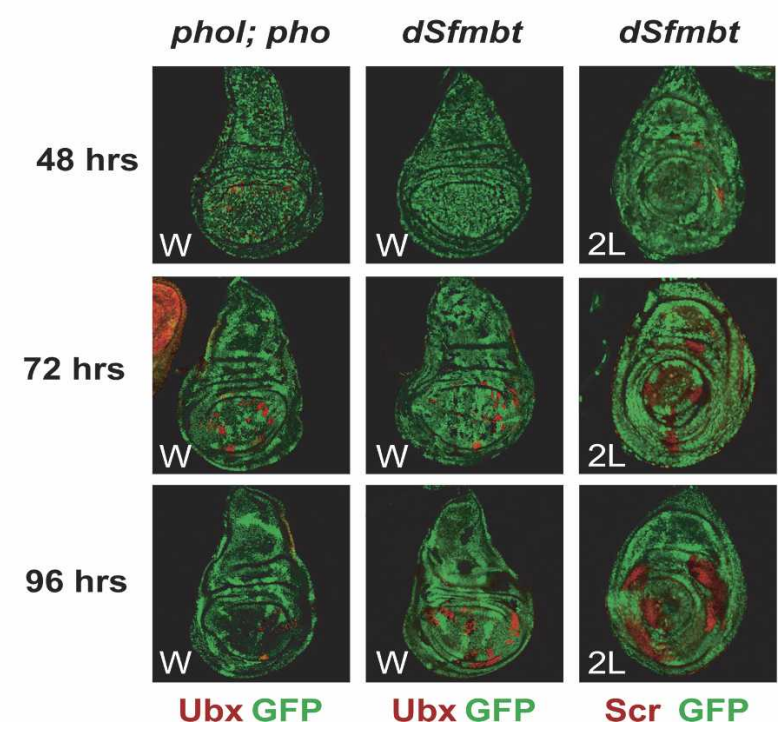

Figure 5. $d S f m b t$ is a novel PcG gene required for HOX gene silencing. (A) Structure of the $d S f m b t^{1}$ knockout allele generated by homologous recombination. In the disruption allele, the whole 3' coding region was inserted in the inverted orientation (see Material and Methods). (B) Imaginal discs with clones of cells that are homozygous for $d S f_{m b} t^{1}$ (dSfmbt) or double homozygous for pho-like $e^{81 A}$ and $p h o^{1}$ (phol; pho) were stained with antibodies against Ubx or Scr (red) and GFP (green); the clones of $d S f m b t$ single-mutant or phol; pho double-mutant cells are marked by the absence of GFP. Discs were analyzed 48, 72 , or $96 \mathrm{~h}$ after clone induction as indicated. (Middle column) Widespread misexpression of Ubx is seen in $d S f m b t$ mutant clones in the wing (W) disc 72 and $96 \mathrm{~h}$ after clone induction; Ubx is normally not expressed in the wing disc. As in the case of other PcG mutants (Beuchle et al. 2001), Ubx is still silenced in some Sfmbt mutant clones (i.e., in the notum), even $96 \mathrm{~h}$ after clone induction. (Right column) Widespread misexpression of Scr is seen in $d S f m b t$ mutant clones in the second leg (2L) disc 72 and $96 \mathrm{~h}$ after clone induction; Scr is normally not expressed in the second leg disc. (Left column) In phol; pho double-mutant clones, misexpression of $\mathrm{Ubx}$ is seen 48 and 72 $\mathrm{h}$ after clone induction. Ninety-six hours after clone induction, most clones of phol; pho double-mutant cells have been eliminated from the disc; note the lack of GFP-negative clones compared with the brightly labeled $G F P^{+} / G F P^{+}$twin spot clones that were induced by the reciprocal recombination event at the time of clone induction. 2L disc images were enlarged; $2 \mathrm{~L}$ discs are in reality half the size of $\mathrm{W}$ discs. expression of HOX genes (Fig. 5B; see also Brown et al. 2003). Removal of dSfmbt and removal of both Pho and Pho-like thus cause a comparable loss of HOX gene silencing (Fig. 5B). This observation is consistent with the idea that dSfmbt is required for repression by both Pho and Pho-like. It should also be noted that $96 \mathrm{~h}$ after clone induction, most pho-like; pho double-mutant clones are eliminated from the disc, suggesting that Pho and Pholike are required for cell proliferation and/or cell viability (Fig. 5B; see also Brown et al. 2003). In contrast, dSfmbt mutant clones do not show any obvious growth defects $96 \mathrm{~h}$ after clone induction (Fig. 5B).

We next wanted to analyze the role of $d S f m b t$ in HOX gene silencing in embryos. In the case of PcG genes, maternally deposited protein product often rescues homozygous mutant embryos to a considerable extent, and, for some PcG loci, their role in HOX gene silencing in embryos can only be fully assessed in homozygous embryos that are derived from mutant germ cells (Struhl 1981; Breen and Duncan 1986; Soto et al. 1995; Birve et al. 2001). dSfmbt ${ }^{1}$ homozygous embryos that are derived from heterozygous mothers show only mild misexpression of HOX genes (see Supplementary Fig. S3). We therefore attempted to generate $d S f m b t^{1}$ homozygous embryos from dSfmbt mutant germ cells. In these experiments, we found that germs cells that lack $d S f m b t$ function fail to develop (for details, see Materials and Methods). dSfmbt thus behaves like the PcG genes E(z) or Su(z)12 (Phillips and Shearn 1990; Birve et al. 2001), both of which are not only required for HOX gene silencing but are also needed for germ cell development. In summary, $d S f m b t$ is a novel PcG gene that is essential for maintenance of HOX gene silencing but is also required for other processes, for example, in the germline.

\section{Transcriptional repression by the dSfmbt protein}

The results described above are consistent with a model in which Pho and Pho-like mediate HOX gene silencing through tethering of dSfmbt to PREs. This scenario predicts that tethering of dSfmbt to DNA by different means might mediate transcriptional repression. Previous studies showed that tethering of the Pc protein to DNA as a fusion protein that contains the DNA-binding domain of Gal4, that is, a Gal4-Pc protein, causes transcriptional repression of reporter genes in Drosophila embryos (Müller 1995). We therefore used the same tethering assay to test whether a Gal4-dSfmbt fusion protein could also represses transcription. The $U A S_{\text {Gal4 }^{-}} U b \mathrm{X}$ $L a c Z$ reporter gene used in these experiments contains five synthetic GAL4-binding sites, the $B X D$ enhancer of $U b x$, and a fragment containing the $U b x$ promoter fused to LacZ (Müller 1995). In transformant embryos that carry this construct, the BXD enhancer activates LacZ expression in a head-to-tail pattern (Fig. 6; cf. Müller 1995). We generated embryos that carry both the $U A S_{\text {Gal4 }}-U b x-L a c Z$ reporter gene and any of the following constructs that allow expression of different effector proteins under the control of a heat-inducible hsp 70 promoter; hs-Gal4-dSfmbt, hs-Gal4-Pc, hs-Gal4, hs-Gal4- 


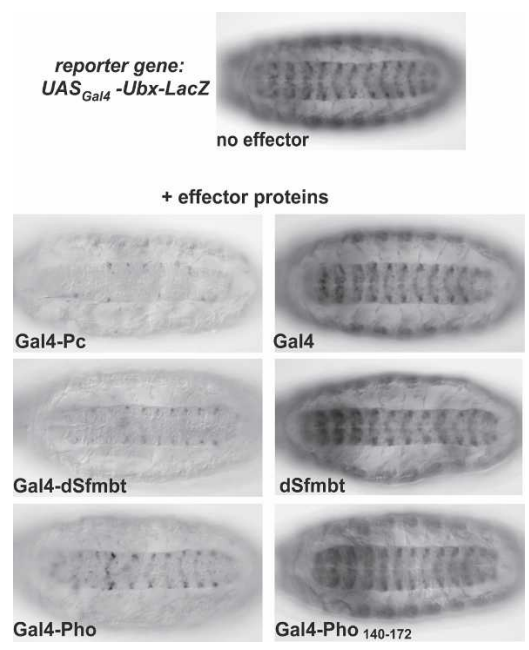

Figure 6. DNA-tethered dSfmbt represses transcription. Ventral views of 14-16-h-old transformant embryos that carry one copy of the $U A S_{\mathrm{Gal}^{-}} \mathrm{Ubx}-\mathrm{LacZ}$ reporter gene (BGUZ construct) (Müller 1995) and one copy of the indicated hsp70-effector construct ("Gal4" corresponds to Gal4 ${ }_{1-147}$, the DNA-binding domain of Gal4); in all cases, embryos were heat-shocked (see Materials and Methods) and stained with antibody against $\beta$-gal. Repression by Gal4-dSfmbt and Gal4-Pho is almost as effective as repression caused by Gal4-Pc protein (Müller 1995). Expression of the Gal4 DNA-binding domain alone or dSfmbt alone does not result in detectable repression. Gal-Pho ${ }_{140-172}$ is also not effective as a repressor in this assay. $\mathrm{Pho}_{140-172}$ includes the so-called spacer domain (Brown et al. 1998) that has been implicated in physical interactions with PRC1 components Pc and Ph (Mohd-Sarip et al. 2002).

Pho, hs-Gal4-Pho ${ }_{140-172}$, or hs-dSfmbt (Fig. 6). In the effector constructs that encode Gal4 fusion proteins, a fragment encoding the DNA-binding domain of Gal4 (i.e., Gal4 ${ }_{1-147}$ ) was fused to the full-length coding region of dSfmbt, Pho, or Pc, respectively, unless otherwise indicated. To assay the silencing capacity of the different effector proteins in embryos, expression of the proteins was induced by repeatedly heat-shocking embryos, starting at the blastoderm stage. As previously described (Müller 1995), the reporter gene was almost completely silenced by the GAL4-Pc fusion protein, whereas the Gal4 DNA-binding domain alone had no effect (Fig. 6). Similarly, GAL4-dSfmbt and Gal4-Pho also caused dramatic down-regulation of the reporter gene. We note, however, that both of these proteins appear to be slightly less potent repressors compared with Gal4-Pc (Fig. 6). Importantly, overexpression of dSfmbt protein alone from the hs-dSfmbt construct had no effect, and the reporter gene was fully active (Fig. 6). Thus, in this assay, $\mathrm{dSfmbt}$ is only effective as a repressor when tethered to the reporter gene by the Gal4 DNA-binding domain. We further note that the Gal4- $\mathrm{Pho}_{140-172}$ fusion protein, containing the "spacer" region of Pho (Brown et al. 1998) that has been implicated in interactions with Pc and Ph in in vitro binding assays (Mohd-Sarip et al. 2002), was ineffective as a repressor (Fig. 6). Taken together, these data show that Gal4-dSfmbt can function as a transcriptional repressor when tethered to DNA.
The MBT repeats of dSfmbt selectively recognize mono- and di-methylated lysine residues in histones H3 and H4

The three-dimensional structures of the human MBTrepeat-containing proteins SCML2 and of L/3)MBT were recently solved. Individual MBT repeats show a $\beta$-barrel structure that most closely resembles the Tudor domain fold (Sathyamurthy et al. 2003; Wang et al. 2003). The structural similarity between MBT repeats, the Tudor domain of SMN, and the chromodomains of HP1 and Pc has led to the proposal that MBT repeats might bind methylated lysine or arginine residues in histone tails (Maurer-Stroh et al. 2003; Sathyamurthy et al. 2003; Wang et al. 2003).

Since the interaction with particular modified histones might play an important role in PcG-mediated repression mechanisms (e.g., Fischle et al. 2003), we used fluorescence polarization assays to measure whether $\mathrm{dSfmbt}$ has histone modification binding activity. A fragment of dSfmbt containing the four MBT repeats (dSfmbt $\left.{ }_{531-980}\right)$ was tested for binding to synthetic $\mathrm{H} 3$ or H4 tail peptides that were either unmodified, or mono-, or di-, or tri-methylated at specific lysine residues. Our fluorescence polarization measurements show that the dSfmbt MBT repeats selectively bind to $\mathrm{H} 3$ tail peptides that are mono- or di-methylated at $\mathrm{K} 9$ and to $\mathrm{H} 4$ tail peptides that are mono- or di-methylated at K20. In contrast, the MBT repeats show far lower ( 20-fold) affinity to the same peptides when tri-methylated at these particular lysine residues (Fig. 7). Indeed, interaction with the tri-methylated $\mathrm{H} 3-\mathrm{K} 9$ and $\mathrm{H} 4-\mathrm{K} 20$ peptides was only slightly stronger than with the corresponding unmodified histone tails. Virtually no binding was observed when the H3 tail peptides were mono-, di-, or tri-methylated at two other known sites of lysine methylation, K4 or K27 (Fig. 7). In addition, no interaction with a H4 peptide acetylated at K16 could be detected. Moreover, interaction with mono-methylated H3-K9 and monomethylated H4-K20 was reduced when the order of amino acids in these peptides was randomly changed. Thus, the MBT repeats of dSfmbt seem to selectively bind to the histone $\mathrm{H} 3$ and $\mathrm{H} 4$ tails when these are either mono- or di-methylated at H3-K9 or H4-K20. The measured dissociation constants for binding to $\mathrm{H} 3-\mathrm{K} 9 \mathrm{mel} / 2$ or $\mathrm{H} 4-\mathrm{K} 20 \mathrm{mel} / 2$ were in the range of $7-12 \mu \mathrm{M}$ (Fig. 7), values that compare well with the affinity of the Pc and HP1 chromodomains for H3 peptides that contain K27me3 and K9me3, respectively (Jacobs and Khorasanizadeh 2002; Fischle et al. 2003). Taken together, these results identify the MBT repeats of dSfmbt as a novel methyl-lysine-binding domain with selectivity for the histone H3-K9 and H4-K20 sites and selectivity for the mono- and di-methylated lysine modification states.

\section{Discussion}

In this study, we show that the PcG protein Pho exists in two stable protein complexes, a Pho-dINO80 complex and PhoRC. Our biochemical and genetic analyses iden- 
A

$$
\begin{aligned}
& \text { H3 (1-15) } \quad \mathrm{H}_{2} \mathrm{~N} \text {-ARTK }{ }_{4} \text { QTARK }{ }_{9} \text { STGGKAY-COOH } \\
& \mathrm{H} 3 \text { (1-15) scrambled } \mathrm{H}_{2} \mathrm{~N} \text {-TAGASRKGKQRKTATY-COOH } \\
& \mathrm{H} 3 \text { (19-35) } \quad \mathrm{H}_{2} \mathrm{~N} \text {-QLATKAARK }{ }_{27} \text { SAPATGGVY-COOH } \\
& \mathrm{H} 4 \text { (12-27) } \quad \mathrm{H}_{2} \mathrm{~N}-\mathrm{KGGAKRHRK}_{20} \text { VLRDNIQ-COOH }
\end{aligned}
$$

B
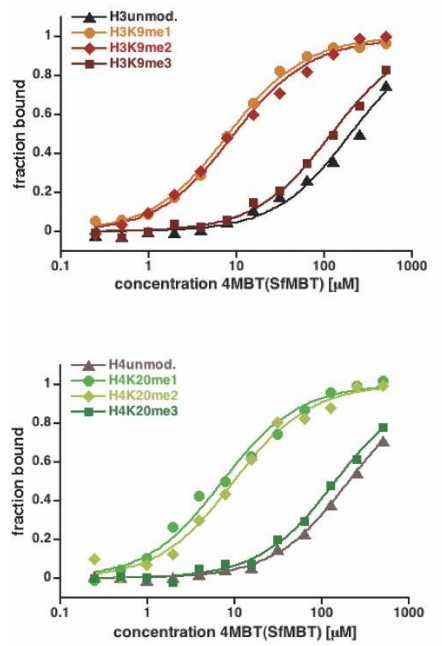

C

$\begin{array}{lc} & \text { Kd } \\ \text { H3 unmod } & 190 \pm 13 \\ \text { H3-K4me1 } & >500 \\ \text { H3-K4me2 } & >500 \\ \text { H3-K4me3 } & >500 \\ \text { H3-K9me1 } & 7 \pm 2 \\ \text { H3-K9me2 } & 9 \pm 2 \\ \text { H3-K9me3 } & 110 \pm 12 \\ \text { H3-K9me1S10ph } & >500 \\ \text { H3-K9me3S10ph } & >500 \\ \text { H3(scram)Kme1 } & 60 \pm 9 \\ & \\ \text { H3 unmod } & 2500 \\ \text { H3-K27me1 } & 180 \pm 20 \\ \text { H3-K27me2 } & 300 \pm 35 \\ \text { H3-K27me3 } & >500 \\ \text { H3-K27me3S10ph } & >500 \\ & \\ \text { H4 unmod } & 200 \pm 25 \\ \text { H4-K20me1 } & 8 \pm 1 \\ \text { H4-K20me2 } & 12 \pm 3 \\ \text { H4-K20me3 } & 130 \pm 15 \\ \text { H4-K16acK20me1 } & 9 \pm 1 \\ \text { H4-K16ac } & 200 \pm 19 \\ \text { H4(scram)Kme1 } & 120 \pm 15 \\ & \end{array}$

Figure 7. Selective binding of dSfmbt MBT repeats to histone $\mathrm{H} 3$ and $\mathrm{H} 4$ tail peptides that carry specific methyl-lysine modifications. (A) Peptide backbones corresponding to different regions of the $\mathrm{H} 3$ and $\mathrm{H} 4 \mathrm{~N}$-terminal tail used in interaction studies. Differentially methylated lysine residues are indicated in red. (B) Binding of the MBT repeats of dSfmbt to H3 peptides that are differentially methylated on $\mathrm{K} 9$ and to $\mathrm{H} 4$ peptides differentially methylated on K20 as measured by fluorescence anisotropy. Data points correspond to averages from three independent experiments. $(C)$ Dissociation constants $(\mathrm{Kd}$ in micromolar, $\mu \mathrm{M}$ ) of the interaction of the MBT repeats of dSfmbt with different modified $\mathrm{H} 3$ and $\mathrm{H} 4$ peptides. Values correspond to averages from at least three independent experiments.

tify PhoRC as a novel PcG protein complex that has a different subunit composition and molecular function than the previously described PcG complexes PRC1 and PRC2. The following conclusions can be drawn from our studies of PhoRC: (1) PhoRC contains Pho and dSfmbt, and these two proteins form a very stable complex that can be purified from embryos and reconstituted from recombinant proteins. (2) PhoRC is bound to PREs in vivo, and PRE-targeting of PhoRC requires intact Pho/Pholike DNA-binding sites. (3) A dSfmbt knockout reveals that dSfmbt is a novel PcG protein that is critically needed for HOX gene silencing. (4) The MBT repeats of dSfmbt are a novel methyl-lysine-recognizing module that selectively binds to the $\mathrm{N}$-terminal tails of histones $\mathrm{H} 3$ and $\mathrm{H} 4$ if they are mono- or di-methylated at $\mathrm{H} 3-\mathrm{K} 9$ or H4-K20, respectively. PhoRC thus contains sequencespecific DNA-binding activity via the Pho protein and methylated histone-binding activity via dSfmbt.

\section{DNA binding of PhoRC at PREs}

Pho and Pho-like are the only PcG proteins with sequence-specific DNA-binding activity (Brown et al. 1998, 2003). Therefore, it is likely that these factors might tether PRC1 or PRC2 to PREs. Unexpectedly, biochemical purification of Pho complexes revealed that Pho exists in stable assemblies with either the PcG protein dSfmbt or components of the Drosophila INO80 complex. However, we failed to purify native or recombinant Pho complexes that contain PRC1 or PRC2 components. Similarly, biochemically purified PRC1 and PRC2 also do not contain Pho (Ng et al. 2000; Saurin et al. 2001; Müller et al. 2002). PhoRC, PRC1, and PRC2 thus seem to be separate biochemical entities.

Reconstitution of recombinant PhoRC shows that dSfmbt binds directly to Pho or to Pho-like to form stable dimeric complexes. Our coimmunoprecipitation assays indicate that such interactions also take place in Drosophila, and we find that dSfmbt is associated with Pho or Pho-like in vivo. Moreover, dSfmbt mutants and pholike; pho double mutants show a comparable loss of HOX gene silencing with similar kinetics. These observations are consistent with dSfmbt being needed for repression by both Pho and Pho-like. Furthermore, the X-ChIP experiments show that Pho/Pho-like DNA-binding sites in PREs are critical for binding of both Pho and dSfmbt at PREs. These data thus suggest that PhoRC is tethered to PREs by Pho or Pho-like.

Binding of the PRC1 subunit Ph at the bxd PRE also depends on intact Pho protein-binding sites. Could dSfmbt in PRE-bound PhoRC interact with Scm or Ph, for example, through the C-terminal SAM domain and thereby tether PRC1 to PREs? In our coimmunoprecipitation experiments, we failed to detect association of dSfmbt with Ph or Scm. These interactions, if they exist, might be either very weak or exist only transiently. Previous studies reported direct physical interactions between Pho and PRC1 or PRC2 subunits, respectively (Mohd-Sarip et al. 2002, 2005; Wang et al. 2004). A possible scenario could therefore be that multiple weak interactions between Pho and dSfmbt with PRC1 and/or with PRC2 subunits might help to stabilize the binding of these complexes to PREs. It is also possible that the lack of Ph binding to the PRE transgene with mutated Pho sites reflects an indirect role of PhoRC that does not involve direct physical interactions between PhoRC and PRC1. In this context, it is worth noting that, on polytene chromosomes, binding of $\mathrm{Ph}$ and other PRC1 components is largely unperturbed in animals that lack both Pho and Pho-like proteins (Brown et al. 2003).

\section{Role of methyl-lysine binding by PhoRC}

Four consecutive MBT repeats are a key feature of the dSfmbt protein. Our fluorescence polarization binding assays suggest that these MBT repeats selectively bind to the $\mathrm{N}$-terminal tail of histones $\mathrm{H} 3$ and $\mathrm{H} 4$ if these are mono- or di-methylated, but not if the same sites are unmethylated or tri-methylated. This novel discrimina- 
tory methyl-lysine-binding activity of MBTs is in stark contrast to the well-documented preference of chromodomains for higher (i.e., tri-) methylated binding sites in histones (Jacobs and Khorasanizadeh 2002; Fischle et al. 2003) and could constitute an important general function of chromatin-associated MBT-containing proteins. The dSfmbt methyl-lysine interaction seems to be specific for the H3K9 and H4K20 methylation sites since matched $\mathrm{H} 3$ peptides that are methylated at different lysine residues (i.e., $\mathrm{H} 3-\mathrm{K} 4 \mathrm{me}$ instead of $\mathrm{H} 3-\mathrm{K} 9 \mathrm{me}$ ) or histone tail peptides in which the methylated lysine residue is embedded in the same amino acid sequence context (i.e., ARKmeS in H3-K27me instead of ARKmeS in H3-K9me) are bound with at least 20-fold lower affinity.

Since our results suggest that dSfmbt is targeted to HOX gene PREs primarily through interaction with Pho (Fig. 3), we reason that binding to methyl-lysine residues in histone tails is not a primary mechanism for targeting dSfmbt to HOX genes. Moreover, recent studies in our laboratory provide evidence that, in the PcG-repressed state, the silenced HOX gene $U b x$ is tri-methylated at $\mathrm{H} 3-\mathrm{K} 9, \mathrm{H} 4-\mathrm{K} 20$, and H3-K27 throughout the gene, whereas lower methylated states of these sites are largely absent (B. Papp and J. Müller, in prep.). What, then, is the role of Sfmbt in binding histones that are mono- or di-methylated at $\mathrm{H} 3-\mathrm{K} 9$ and H4-K20 in silenced HOX genes? Mono- and di-methylation of H4K20 are very abundant modifications in Drosophila chromatin (Nishioka et al. 2002; Bonaldi et al. 2004; Karachentsev et al. 2005), and mass spectroscopic analyses of histones in embryos imply that lower methylated forms of histone $\mathrm{H} 4$ (i.e., H4-K20me2) already exist prior to becoming incorporated into chromatin during $S$ phase (Bonaldi et al. 2004). It is therefore tempting to speculate that dSfmbt, tethered to PREs by Pho, scans the flanking HOX gene chromatin for nucleosomes that are only mono- or di-methylated at H3-K9 or H4-K20 and docks onto such nucleosomes through its MBT repeats. We hypothesize that through this bridging interaction, nucleosomes of lower methylated states might be brought into proximity to PRE-bound PRC2 (Cao et al. 2002) and other currently unknown HMTases that are responsible for local tri-methylation of $\mathrm{H} 3-\mathrm{K} 9$ and $\mathrm{H} 4-\mathrm{K} 20$ in silenced HOX genes. According to this model, PRE-bound PhoRC would act as a "grappling hook" that tethers mono- and di-methylated histones in silenced HOX gene chromatin to PREs to ensure that they become hypermethylated to the tri-methylated state. Such a chromatin-scanning function might be particularly important during S phase, when newly incorporated histone octamers need to become fully tri-methylated in order to maintain silencing of HOX genes.

\section{Materials and methods}

\section{TAP}

The $\alpha$-tubulin-Pho-TAP transgene in the Drosophila transformation vector CaSpeR has the following structure: a 2.6-kb fragment of the $\alpha$-tubulin 1 gene containing promoter and $5^{\prime}$ un- translated region sequences (Struhl and Basler 1993) linked to a pho cDNA fragment that contains the whole Pho ORF $\left(\mathrm{Pho}_{1-520}\right)$; the termination codon was replaced by the sequence GGC (Gly) to fuse it in-frame to the C-terminal TAP tag (detailed plasmid map available upon request). Rescue function of the $\alpha$-tubulin-Pho-TAP transgene was tested by introducing the transgene into a $p h o^{1} / \mathrm{ci}^{D}$ mutant background; with four different transgene inserts, we obtained $w$; $\alpha$-tubulin-Pho-TAP; pho $^{1} /$ pho ${ }^{1}$ animals that were wild type in appearance and fully viable and fertile. TAP was performed on embryonic nuclear extracts from wild-type and $\alpha$-tubulin-Pho-TAP transgenic embryos (a detailed protocol for extract preparation and purification is available as Supplemental Material S4).

\section{Mass spectrometry}

A detailed list of peptide sequences obtained from mass spectrometry analysis of the protein bands shown in Figure 1 is available in Supplemental Material S1.

\section{Antibody production}

Antibodies against $\mathrm{Pho}_{2-382}, \mathrm{dSfmbt}_{531-980}, \mathrm{dSfmbt}_{1130-1220}$, dINO80 $_{16-310}$, and dINO80 $1261-1510$ were raised in rabbits. In all cases, epitopes for antibody production were expressed as 6xHis-tagged fusion proteins in Escherichia coli.

\section{Glycerol gradient sedimentation analysis}

Embryonic nuclear extract $(0.5 \mathrm{~mL})$ was directly loaded onto 12 $\mathrm{mL}$ of $20 \%-50 \%$ glycerol gradients in $15 \mathrm{mM}$ HEPES (pH 7.9), $200 \mathrm{mM} \mathrm{KCl}, 1.5 \mathrm{mM} \mathrm{MgCl} 2,0.2 \mathrm{mM}$ EDTA (pH 7.9), and 1 mM DTT. Sedimentation was done by centrifugation for $40 \mathrm{~h}$ at $38,000 \mathrm{rpm}$ in a Beckman SW40 rotor at $4^{\circ} \mathrm{C}$. Fractions $(500 \mu \mathrm{L})$ were collected and analyzed by Western blot.

\section{Immunoprecipitations}

Three-hundred micrograms of crude embryonic nuclear extract (see Supplemental Material S4) was incubated with 2-10 $\mu \mathrm{L}$ of the appropriate antibody for $2 \mathrm{~h}$ at $4^{\circ} \mathrm{C}$. Immunoprecipitates were recovered with protein A-agarose beads (Roche) and washed five times with NE200 (15 mM HEPES at pH 7.9, 20\% glycerol, $1.5 \mathrm{mM} \mathrm{MgCl}_{2}, 200 \mathrm{mM} \mathrm{KCl}, 0.2 \mathrm{mM}$ EDTA at pH 7.9, $1 \mathrm{mM}$ DTT) containing $0.1 \%$ Tween 20 , and proteins were eluted with SDS sample buffer.

\section{X-ChIP assays}

$\mathrm{X}$-ChIP on $P R E_{D}$ (line $5 \mathrm{~B}$ ) and $P R E_{D}$ Pho mut (line 7.4) transformant embryos (Fritsch et al. 1999) was done as described in Supplemental Material S4; the primers used for amplification are listed in Supplemental Material S5.

\section{Protein expression and purification}

Baculoviruses expressing Flag-Esc, Flag-E(z), E(z), and Flag-Pc have been described previously (Francis et al. 2001; Müller et al. 2002). Viruses expressing Pho-Flag, Pho-like-Flag, Flag-dSfmbt, dSfmbt, Flag-Reptin, Reptin, Flag-Pontin, and Pontin were generated for this study; in all cases, the whole ORF was subcloned into pFastBac (plasmid maps are available upon request). Flagaffinity purification of complexes was carried out as described (Müller et al. 2002). 


\section{dSfmbt knockout and characterization of dSfmbt phenotype}

An ends-out recombination strategy was used to disrupt $d S f m b t$ and replace part of it with a miniwhite marker gene following the strategy described (Gong and Golic 2003). A dSfmbt disruption construct was generated by cloning $3.3 \mathrm{~kb}$ of $5^{\prime}$-flanking and coding sequence (AE003639 nucleotides 53,047-56,375) between the NotI and SphI sites and $3.3 \mathrm{~kb}$ of dSfmbt 3' coding sequence (AE003639 nucleotides 49,756-52,990) into the BamHI site of pw35 (Gong and Golic 2003). Inadvertently, dSfmbt $3^{\prime}$ coding sequences were cloned in the $5^{\prime} \rightarrow 3^{\prime}$ orientation with respect to miniwhite in pw35, and the isolated $d S$ $f m b t^{1}$ allele contains not only replacement of AE nucleotides $52,991-53,045$ by the miniwhite disruption cassette, but it also carries an inversion and partial duplication of $3^{\prime}$ dSfmbt coding sequences. PCR followed by sequence analysis was used to characterize the disruption of the $d S f m b t$ coding region in animals that were homozygous for the isolated $d S f m b t^{1}$ allele, and the integrity of sequences flanking the neighboring CG5216 and CG5439 genes was also analyzed by PCR. Lack of a dSfmbt signal in $d S f m b t^{1}$ mutant clones in imaginal discs suggests that $d S f m b t^{1}$ is a protein-null allele (Supplementary Fig. S2).

$d S f m b t^{1}$ homozygotes and $d S f m b t^{1} / D f(2 L) B S C 30$ transheterozygotes die as wandering third instar larvae; a small fraction develops into highly abnormal prepupae that fail to develop further. At $25^{\circ} \mathrm{C}, d S f m b t^{1}$ homozygotes and $d S f m b t^{1} /$ $D f(2 L) B S C 30$ transheterozygotes reach the expected body length $120 \mathrm{~h} \mathrm{AEL}$, but their discs are very small. Many of these animals continue to grow as wandering larvae, and their discs eventually develop into amorphous tumor-like tissue; misexpression of Ubx is observed in cells in the CNS and in discs. Clonal analysis: $d S f m b t^{1}$ mutant clones in imaginal discs were generated and analyzed by antibody staining as described (Beuchle et al. 2001). Germline clones were induced with the Flp ovo ${ }^{D}$ system, but no eggs were recovered, and analysis of ovaries from females with $d S f m b t^{1}$ mutant germ cells suggests that lack of dSfmbt results in developmental arrest during oogenesis.

\section{Silencing by Gal4-PcG fusion proteins in embryos}

Transgenic Drosophila strains carrying the hsp70-Gal-Pc and the BGUZ reporter genes have been described (Müller 1995). Transformants carrying the following effector constructs were generated for this study: hsp70-Gal4 ${ }_{1-147}-d S f m b t_{1-1220}$, hsp 70dSfmbt ${ }_{1-1220,}$ hsp70-Gal4 ${ }_{1-147}$, hsp70-Gal4 ${ }_{1-147}-\mathrm{PhO}_{2-520}$, and hsp70-Gal4 1-147 $-\mathrm{Pho}_{140-172}$ (detailed plasmid maps are available upon request). In all cases, embryos carried one copy of the $B G U Z$ reporter gene and one copy of an hsp70-effector transgene; in some cases, control embryos carrying only $B G U Z$ were "spiked in" to calibrate the HRP staining reactions. In all cases, embryos were collected for $2 \mathrm{~h}$ and were repeatedly heatshocked $\left(20 \mathrm{~min}\right.$ at $\left.37^{\circ} \mathrm{C}\right)$ every $2 \mathrm{~h}$, starting at $2-4 \mathrm{~h}$ of development. Embryos were fixed and stained with antibody against $\beta$-gal as described (Müller 1995).

\section{Fluorescence anisotropy measurements}

Peptides corresponding to the $\mathrm{N}$ terminus of $\mathrm{H} 3$ and $\mathrm{H} 4$ either unmodified or containing mono-, di-, or tri-methylated lysine residues were synthesized by the Rockefeller Proteomics Core Facility. Peptides were either N-terminally labeled with succinimidyl-fluorescein while still protected or after cleavage from the resin and deprotection. All labeled peptides were HPLCpurified by reversed phase chromatography, and identity was verified by mass spectrometry. The four MBT repeats of $\mathrm{dSfmbt}$ were fused to an $\mathrm{N}$-terminal His tag (6xHis-dSfmbt $\left.{ }_{531-980}\right)$. Fusion protein was expressed in E. coli and purified by Ni-affinity and gel filtration chromatography (Sephadex 75). Fluorescence polarization binding assays were performed under conditions of $20 \mathrm{mM}$ imidazole ( $\mathrm{pH} 8.0$ ), $25 \mathrm{mM} \mathrm{NaCl}$, and $2 \mathrm{mM} \mathrm{DTT}$ and in the presence of $100 \mathrm{nM}$ fluorescein-labeled peptide following a previously described protocol (Jacobs et al. 2004).

\section{Acknowledgments}

We thank Hugh Brock, Danny Brower, Judith Kassis, Bob Kingston, Marie Meister, Jacques Pradel, Jeff Simon, and Peter Verrijzer for reagents. W.F. thanks David Allis for support while part of this work was carried out in his laboratory; W.F. was a Robert Black Fellow of the Damon Runyon Cancer Research Foundation. We thank Aditya Sengupta and the LAR at EMBL for help with raising antibodies, Ann-Mari Voie for help with generating transgenic fly strains, and Vladimir Benes at the EMBL Gene Core Facility for excellent support. We thank Asifa Akhtar and Andreas Ladurner for discussions. Comments from Elisa Izaurralde helped to improve the manuscript. C.F., T.K., and B.P. were supported by grants from the DFG.

\section{References}

Bauer, A., Chauvet, S., Huber, O., Usseglio, F., Rothbacher, U., Aragnol, D., Kemler, R., and Pradel, J. 2000. Pontin52 and reptin52 function as antagonistic regulators of $\beta$-catenin signalling activity. $E M B O T$. 19: 6121-6130.

Beuchle, D., Struhl, G., and Muller, J. 2001. Polycomb group proteins and heritable silencing of Drosophila Hox genes. Development 128: 993-1004.

Birve, A., Sengupta, A.K., Beuchle, D., Larsson, J., Kennison, J.A., Rasmuson-Lestander, A., and Müller, J. 2001. Su(z)12, a novel Drosophila Polycomb group gene that is conserved in vertebrates and plants. Development 128: 3371-3379.

Bonaldi, T., Imhof, A., and Regula, J.T. 2004. A combination of different mass spectroscopic techniques for the analysis of dynamic changes of histone modifications. Proteomics 4: 1382-1396.

Breen, T.R. and Duncan, I.M. 1986. Maternal expression of genes that regulate the bithorax complex of Drosophila melanogaster. Dev. Biol. 118: 442-456.

Brown, J.L., Mucci, D., Whiteley, M., Dirksen, M.L., and Kassis, J.A. 1998. The Drosophila Polycomb group gene pleiohomeotic encodes a DNA binding protein with homology to the transcription factor YY1. Mol. Cell 1: 1057-1064.

Brown, J.L., Fritsch, C., Muller, J., and Kassis, J.A. 2003. The Drosophila pho-like gene encodes a YY1-related DNA binding protein that is redundant with pleiohomeotic in homeotic gene silencing. Development 130: 285-294.

Busturia, A., Lloyd, A., Bejarano, F., Zavortink, M., Xin, H., and Sakonju, S. 2001. The MCP silencer of the Drosophila Abd-B gene requires both Pleiohomeotic and GAGA factor for the maintenance of repression. Development 128: 2163-2173.

Cao, R., Wang, L., Wang, H., Xia, L., Erdjument-Bromage, H., Tempst, P., Jones, R.S., and Zhang, Y. 2002. Role of histone H3 lysine 27 methylation in Polycomb-group silencing. Science 298: 1039-1043.

Chan, C.S., Rastelli, L., and Pirrotta, V. 1994. A Polycomb response element in the Ubx gene that determines an epigenetically inherited state of repression. EMBO J. 13: 25532564.

Czermin, B., Melfi, R., McCabe, D., Seitz, V., Imhof, A., and 
Pirrotta, V. 2002. Drosophila Enhancer of Zeste/ESC complexes have a histone $\mathrm{H} 3$ methyltransferase activity that marks chromosomal Polycomb sites. Cell 111: 185-196.

Fischle, W., Wang, Y., Jacobs, S.A., Kim, Y., Allis, C.D., and Khorasanizadeh, S. 2003. Molecular basis for the discrimination of repressive methyl-lysine marks in histone $\mathrm{H} 3$ by Polycomb and HP1 chromodomains. Genes \& Dev. 17: 1870-1881.

Francis, N.J. and Kingston, R.E. 2001. Mechanisms of transcriptional memory. Nat. Rev. Mol. Cell Biol. 2: 409-421.

Francis, N.J., Saurin, A.J., Shao, Z., and Kingston, R.E. 2001. Reconstitution of a functional core polycomb repressive complex. Mol. Cell 8: 545-556.

Fritsch, C., Brown, J.L., Kassis, J.A., and Muller, J. 1999. The DNA-binding Polycomb group protein pleiohomeotic mediates silencing of a Drosophila homeotic gene. Development 126: 3905-3913.

Gong, W.J. and Golic, K.G. 2003. Ends-out, or replacement, gene targeting in Drosophila. Proc. Natl. Acad. Sci. 100: 25562561.

Hagstrom, K., Muller, M., and Schedl, P. 1997. A Polycomb and GAGA dependent silencer adjoins the Fab-7 boundary in the Drosophila bithorax complex. Genetics 146: 1365-1380.

Jacobs, S.A. and Khorasanizadeh, S. 2002. Structure of HP1 chromodomain bound to a lysine 9-methylated histone $\mathrm{H} 3$ tail. Science 295: 2080-2083.

Jacobs, S.A., Fischle, W., and Khorasanizadeh, S. 2004. Assays for the determination of structure and dynamics of the interaction of the chromodomain with histone peptides. Methods Enzymol. 376: 131-148.

Jonsson, K.O., Jha, S., Wohlschlegel, J.A., and Dutta, A. 2004. Rvb1p/Rvb2p recruit Arp5p and assemble a functional Ino80 chromatin remodeling complex. Mol. Cell 16: 465-477.

Karachentsev, D., Sarma, K., Reinberg, D., and Steward, R. 2005. PR-Set7-dependent methylation of histone H4 Lys 20 functions in repression of gene expression and is essential for mitosis. Genes \& Dev. 19: 431-435.

Ketel, C.S., Andersen, E.F., Vargas, M.L., Suh, J., Strome, S., and Simon, J.A. 2005. Subunit contributions to histone methyltransferase activities of fly and worm Polycomb group complexes. Mol. Cell. Biol. 25: 6857-6868.

Maurer-Stroh, S., Dickens, N.J., Hughes-Davies, L., Kouzarides, T., Eisenhaber, F., and Ponting, C.P. 2003. The Tudor domain 'Royal Family': Tudor, plant Agenet, Chromo, PWWP and MBT domains. Trends Biochem. Sci. 28: 69-74.

Mihaly, J., Mishra, R.K., and Karch, F. 1998. A conserved sequence motif in Polycomb-response elements. Mol. Cell 1: 1065-1066.

Mishra, R.K., Mihaly, J., Barges, S., Spierer, A., Karch, F., Hagstrom, K., Schweinsberg, S.E., and Schedl, P. 2001. The iab-7 Polycomb response element maps to a nucleosome-free region of chromatin and requires both GAGA and pleiohomeotic for silencing activity. Mol. Cell. Biol. 21: 1311-1318.

Mohd-Sarip, A., Venturini, F., Chalkley, G.E., and Verrijzer, C.P. 2002. Pleiohomeotic can link Polycomb to DNA and mediate transcriptional repression. Mol. Cell. Biol. 22: 74737483.

Mohd-Sarip, A., Cleard, F., Mishra, R.K., Karch, F., and Verrijzer, C.P. 2005. Synergistic recognition of an epigenetic DNA element by Pleiohomeotic and a Polycomb core complex. Genes \& Dev. 19: 1755-1760.

Müller, J. 1995. Transcriptional silencing by the Polycomb protein in Drosophila embryos. EMBO J. 14: 1209-1220.

Müller, J., Hart, C.M., Francis, N.J., Vargas, M.L., Sengupta, A., Wild, B., Miller, E.L., O'Connor, M.B., Kingston, R.E., and Simon, J.A. 2002. Histone methyltransferase activity of a
Drosophila Polycomb group repressor complex. Cell 111: 197-208.

Nekrasov, M., Wild, B., and Müller, J. 2005. Nucleosome binding and histone methyltransferase activity of Drosophila PRC2. EMBO Rep. 6: 348-353.

Ng, J., Hart, C.M., Morgan, K., and Simon, J.A. 2000. A Drosophila ESC-E(Z) protein complex is distinct from other Polycomb group complexes and contains covalently modified ESC. Mol. Cell. Biol. 20: 3069-3078.

Nishioka, K., Rice, J.C., Sarma, K., Erdjument-Bromage, H., Werner, J., Wang, Y., Chuikov, S., Valenzuela, P., Tempst, P., Steward, R., et al. 2002. PR-Set7 is a nucleosome-specific methyltransferase that modifies lysine 20 of histone $\mathrm{H} 4$ and is associated with silent chromatin. Mol. Cell 9: 1201-1213.

Orlando, V., Jane, E.P., Chinwalla, V., Harte, P.J., and Paro, R. 1998. Binding of trithorax and Polycomb proteins to the bithorax complex: Dynamic changes during early Drosophila embryogenesis. EMBO J. 17: 5141-5150.

Peterson, A.J., Kyba, M., Bornemann, D., Morgan, K., Brock, H.W., and Simon, J. 1997. A domain shared by the Polycomb group proteins $\mathrm{Scm}$ and ph mediates heterotypic and homotypic interactions. Mol. Cell. Biol. 17: 6683-6692.

Peterson, A.J., Mallin, D.R., Francis, N.J., Ketel, C.S., Stamm, J., Voeller, R.K., Kingston, R.E., and Simon, J.A. 2004. Requirement for Sex comb on midleg protein interactions in Drosophila Polycomb group repression. Genetics 167: 12251239.

Phillips, M.D. and Shearn, A. 1990. Mutations in polycombeotic, a Drosophila Polycomb-group gene, cause a wide range of maternal and zygotic phenotypes. Genetics 125: 91-101.

Poux, S., Melfi, R., and Pirrotta, V. 2001. Establishment of Polycomb silencing requires a transient interaction between PC and ESC. Genes \& Dev. 15: 2509-2514.

Rigaut, G., Shevchenko, A., Rutz, B., Wilm, M., Mann, M., and Seraphin, B. 1999. A generic protein purification method for protein complex characterization and proteome exploration. Nat. Biotechnol. 17: 1030-1032.

Ringrose, L. and Paro, R. 2004. Epigenetic regulation of cellular memory by the Polycomb and Trithorax group proteins. Annu. Rev. Genet. 38: 413-443.

Ringrose, L., Rehmsmeier, M., Dura, J.M., and Paro, R. 2003. Genome-wide prediction of Polycomb/Trithorax response elements in Drosophila melanogaster. Dev. Cell 5: 759-771.

Sathyamurthy, A., Allen, M.D., Murzin, A.G., and Bycroft, M. 2003. Structure of the malignant brain tumor (MBT) repeats in Sex Comb on Midleg-like 2 (SCML2). J. Biol. Chem. 278: 46968-46973.

Saurin, A.J., Shao, Z., Erdjument-Bromage, H., Tempst, P., and Kingston, R.E. 2001. A Drosophila Polycomb group complex includes Zeste and dTAFII proteins. Nature 412: 655-660.

Sengupta, A.K., Kuhrs, A., and Müller, J. 2004. General transcriptional silencing by a Polycomb response element in Drosophila. Development 131: 1959-1965.

Shao, Z., Raible, F., Mollaaghababa, R., Guyon, J.R., Wu, C.T., Bender, W., and Kingston, R.E. 1999. Stabilization of chromatin structure by PRC1, a Polycomb complex. Cell 98: 3746.

Shen, X., Mizuguchi, G., Hamiche, A., and Wu, C. 2000. A chromatin remodelling complex involved in transcription and DNA processing. Nature 406: 541-544.

Shimell, M.J., Peterson, A.J., Burr, J., Simon, J.A., and O'Connor, M.B. 2000. Functional analysis of repressor binding sites in the iab-2 regulatory region of the abdominal-A homeotic gene. Dev. Biol. 218: 38-52.

Soto, M.C., Chou, T.B., and Bender, W. 1995. Comparison of germline mosaics of genes in the Polycomb group of Dro- 
Klymenko et al.

sophila melanogaster. Genetics 140: 231-243.

Struhl, G. 1981. A gene product required for correct initiation of segmental determination in Drosophila. Nature 293: 36-41.

Struhl, G. and Basler, K. 1993. Organizing activity of wingless protein in Drosophila. Cell 72: 527-540.

Strutt, H. and Paro, R. 1997. The Polycomb protein complex of Drosophila has differential composition at different target genes. Mol. Cell. Biol. 17: 6773-6783.

Tie, F., Prasad-Sinha, J., Birve, A., Rasmuson-Lestander, A., and Harte, P.J. 2003. A 1-megadalton ESC/E(Z) complex from Drosophila that contains polycomblike and RPD3. Mol. Cell. Biol. 23: 3352-3362.

Usui, H., Ichikawa, T., Kobayashi, K., and Kumanishi, T. 2000. Cloning of a novel murine gene Sfmbt, Scm-related gene containing four mbt domains, structurally belonging to the Polycomb group of genes. Gene 248: 127-135.

Wang, W.K., Tereshko, V., Boccuni, P., MacGrogan, D., Nimer, S.D., and Patel, D.J. 2003. Malignant brain tumor repeats: A three-leaved propeller architecture with ligand/peptide binding pockets. Structure 11: 775-789.

Wang, L., Brown, J.L., Cao, R., Zhang, Y., Kassis, J.A., and Jones, R.S. 2004. Hierarchical recruitment of Polycomb group silencing complexes. Mol. Cell 14: 637-646.

Zink, D. and Paro, R. 1995. Drosophila Polycomb-group regulated chromatin inhibits the accessibility of a trans-activator to its target DNA. EMBO J. 14: 5660-5671. 


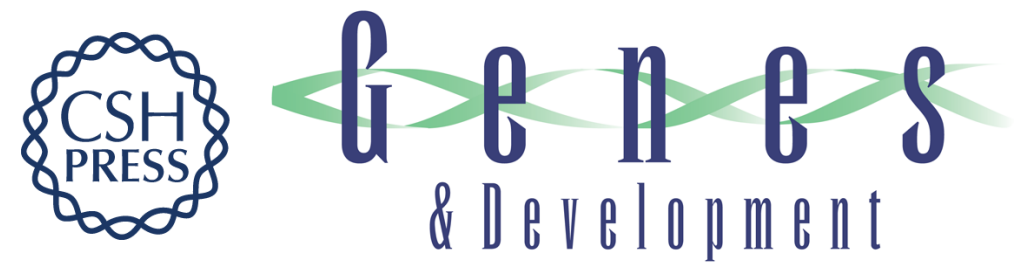

\section{A Polycomb group protein complex with sequence-specific DNA-binding and selective methyl-lysine-binding activities}

Tetyana Klymenko, Bernadett Papp, Wolfgang Fischle, et al.

Genes Dev. 2006, 20:

Access the most recent version at doi:10.1101/gad.377406

\section{Supplemental http://genesdev.cshlp.org/content/suppl/2006/04/17/gad.377406.DC1 Material}

References This article cites 55 articles, 26 of which can be accessed free at: http://genesdev.cshlp.org/content/20/9/1110.full.html\#ref-list-1

\section{License}

Email Alerting

Receive free email alerts when new articles cite this article - sign up in the box at the top Service

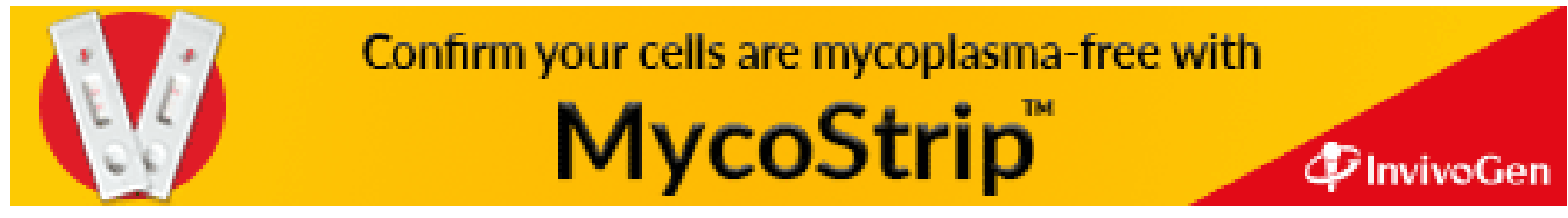

